\title{
Molecular Insights into Error-Prone DNA Replication and Error-Free Lesion Bypass
}

\author{
Andreas Marx* and Daniel Summerer ${ }^{[a]}$
}

\section{KEYWORDS:}

DNA damage DNA polymerases - DNA replication - thymidine dimers - UV light

Ultraviolet (UV) light causes a variety of damage in DNA. The most abundant lesions are pyrimidine dimers such as the pyrimidine (6-4) pyrimidone photoproduct (6-4PP) and the cis,syn cyclobutane-pyrimidine dimer (CPD) $)^{[1]}$ (Scheme 1). These lesions are often repaired sluggishly and remain in the DNA,

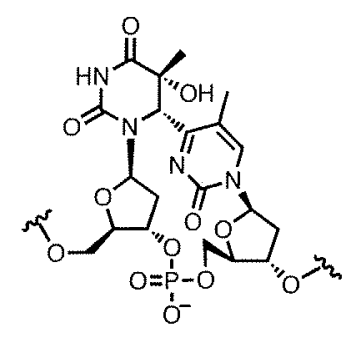

6-4PP

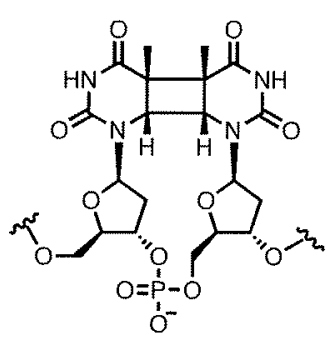

CPD
Scheme 1. UV-light-induced thymidine dimer DNA lesions $6-4 P P=$ pyrimidine (6-4) pyrimidone photoproduct, $C P D=$ cis,syn cyclobutane pyrimidine dimer.

where they considerably impede the DNA replication machinery." How cells perform DNA synthesis past these kind of lesions has for a long time remained obscure. Only recently, it has been discovered that several specialized DNA polymerases which belong to the new superfamily $Y$ are clearly involved in translesion synthesis. ${ }^{[2]}$ These DNA polymerases (pols) are found in a variety of organisms ranging from Escherischia coli to humans. One of their most prominent functional characteristics is their high

[a] Dr. A. Marx, Dipl.-Chem. D. Summerer Kekulé-Institut für Organische Chemie und Biochemie

Universität Bonn

Gerhard-Domagk-Strasse

53121 Bonn (Germany)

Fax: $(+49) 228-73-5388$

E-mail:a.marx@uni-bonn.de error propensity when dealing with undamaged DNA, which distinguishes them from known high-fidelity DNA polymerases. Most of their biological roles and functions have not been elucidated yet. Nevertheless, recent investigations suggest the involvement of pol $\iota$ together with pol $\zeta$ in error-prone translesion DNA synthesis. ${ }^{[3]}$ Furthermore, it has been shown that pol ı possesses lyase activity, which suggests that the enzyme may be involved in DNA repair processes. ${ }^{[4]}$ DNA polymerase $\eta$ is unique among the eukaryotic polymerases since it has the ability to perform error-free bypass synthesis through CPD lesions, which are poorly removed by repair processes. ${ }^{[5]}$ It has been shown that cells from patients with the inherited disorder of a variant form of xeroderma pigmentosum (XP-V) are unable to perform CPD bypass synthesis as a result of expression of severely truncated or functionally inactive forms of pol $\eta{ }^{\left[{ }^{[5 e}, f\right]}$ These observations strongly suggest that pol $\eta$ is the XP-V gene product and that the polymerase is involved in suppression of sunlight-induced skin cancer. Yeast and human pol $\eta$ replicate through a cis,syn thymidine - thymidine dimer by inserting two deoxyadenosine residues opposite the lesion. Although, pol $\eta$ misinserts noncomplementary nucleotides opposite the first thymidine (T) in the lesion with high frequency, extension from mismatched substrates is greatly impeded. Presumably, this feature allows the polymerase to dissociate from the primer template complex and recruit exonucleases to remove the mispaired nucleotide. Overall, the UV-damaged lesion would be more selectively bypassed than predicted from the misinsertion frequency alone. In general, pol $\eta$ appears to be able to replicate DNA accurately through the lesions that are most frequently formed as a result of exposure to UV light.

These unique properties of pol $\eta$ instantly triggered interest in its molecular and structural basis. Recently, several crystal structures of error-prone Y-family DNA polymerases have been solved from which valuable new insights into their molecular action can be drawn. ${ }^{[6]}$ Trincao et al. determined the crystal structure of an $\mathrm{N}$-terminal fragment of Saccharomyces cerevisiae pol $\eta$ that includes the active site. ${ }^{[6]}$ This structure reveals that pol $\eta$ retains the overall shape of a right hand composed of thumb, palm, and finger domains, as seen in other known DNA polymerases ${ }^{[6-9]}$ (Figure 1). However, pol $\eta$ contains a novel polymerase-associated domain (PAD), which mimics an extra set of fingers. The structure shows that the palm domain of pol $\eta$ is nearly superimposable with that of high-fidelity DNA polymerases. ${ }^{[7,8]}$ In contrast, the finger and thumb domains, which make extensive contacts with the primer template and incoming triphosphate in other DNA polymerases, are unusually small and stubby. In particular, the "O helices", which are believed to play a pivotal role in fidelity checking, are absent in pol $\eta$. Further insights into error-prone DNA synthesis were gained through the crystal structures of Sulfolobus solfataricus P2 DNA polymerase IV (Dpo4) reported by Ling et al. ${ }^{(6 d)}$ This enzyme is able to bypass cis-syn CPD lesions efficiently and thus, with respect to translesion synthesis, the enzymatic properties of Dpo4 are similar to that of the distantly related eukaryotic pol $\eta$. Ling et al. successfully obtained Dpo4 structures in ternary complexes with the DNA primer template and either a canonical or noncanonical incoming nucleoside triphosphate. The overall structure of the Dpo4-DNA ternary complex showed the familiar shape of a half- 


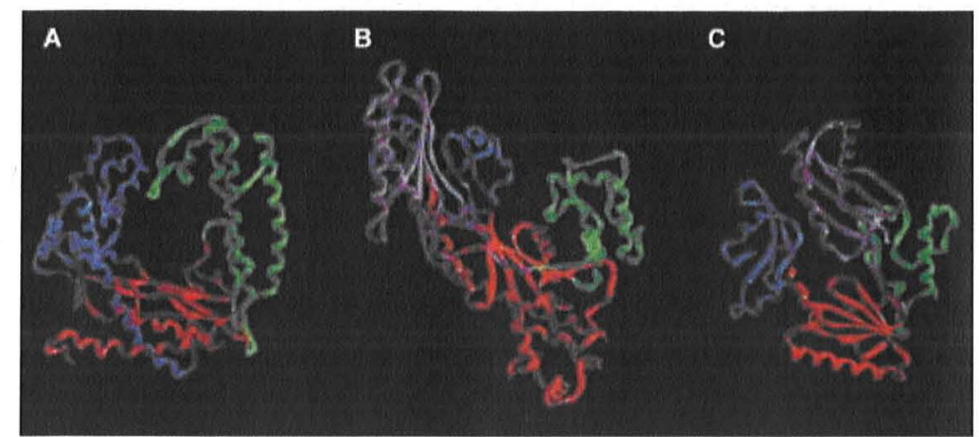

Figure 1. DNA polymerase structures. A: ternary complex of Taq DNA polymerase (Protein Data Bank (PDB) entry code $2 K T Q$ ); $B$ : DNA polymerase $\eta$ (coordinates provided by the authors); $C$ : the ternary complex of Dpo 4 (PDB entry code 1JX4). The palm (red), fingers (blue), thumb (green), and polymerase-associated domain/little finger (purple) are shown.

open right hand found in several DNA polymerases in which parts of the primer template are embedded. ${ }^{[7,8]}$ Analogous to pol $\eta$, Dpo4 contains a domain termed the little finger in addition to the finger, palm, and thumb domains. Again, the palm domain is structurally similar to those of other DNA polymerases and the essential carboxylates in Dpo4 are in identical positions to those in T7, Klenow, Bacillus, Taq, and RB69 DNA polymerases, as well as in HIV reverse transcriptase. ${ }^{[8]}$ However, the finger and thumb domains of Dpo4 that surround the incoming triphosphate and template nucleotide are unusually small. The $\mathrm{O}$ helices that are believed to be involved in selection for the right nucleobase geometry and are present in all high-fidelity DNA polymerases are absent in Dpo4 as well as in pol $\eta{ }^{[7]}$ Overall, the nascent base pair between the template and incoming nucleotide in Dpo4 is less tightly surrounded in the vicinity of the enzyme than it is in highfidelity DNA polymerases. The open and solvent-accessible active site may be one structural basis for the error-prone replication of undamaged DNA by Y-family DNA polymerases. These findings further support the current concept that geometrical constraints are the major causative of DNA polymerase fidelity. ${ }^{[10]}$

The primer template complexed by Dpo4 is nearly standard B-form DNA and undergoes protein interactions with eight base pairs through both hydrogen bonds and van der Waals contacts. These interactions are mostly with the phosphodiester and sugar moieties. In contrast to highfidelity DNA polymerases, no hydrogen bonds with donor or acceptor sites of the nucleobases through the minor groove are observed in Dpo4. Checking of hydrogen-bonding patterns by high-fidelity DNA polymerases is believed to contribute to fidelity through prevention of inadvertent sealing of mismatched base pairs into the nascent DNA. ${ }^{[7 c, 10]}$

The second crystal structure obtained by Ling et al. contains a mismatched dideoxyguanosine triphosphate (ddGTP) in the active site of Dpo4. ${ }^{[6 \mathrm{~d}]}$ In the active site, conformations of the sugar phosphate moieties of the primer, template, and nucleoside triphosphate differ significantly from that found when a canonical nucleotide is bound. Translocation of the template without replication of the first template base (guanine) leads the incoming ddGTP to form a canonical base pair with the next template base (cytosine; Figure 2). This structure shows that the

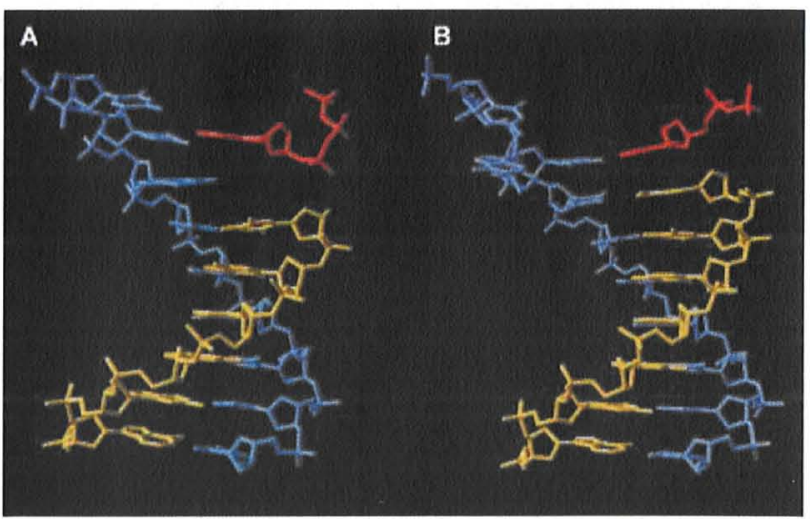

Figure 2. DNA bound in the active site. Protein elements are removed for clarity (PDB entry codes 1 JX4 and 1JXL). A: primer (gold) and template (blue) in Dpo4 with incoming noncanonical triphosphate (ddGTP, red) exhibiting translocation of the template that results in a base pair between ddGTP and the second nucleobase in the template. B: primer (gold) and template (blue) in Dpo4 with incoming canonical triphosphate ddATP (red). 
We thank A.K. Aggarwal, New York for kindly providing the coordinates of DNA polymerase $\eta$ and $M$. Strerath Bonn for his assistance in preparing the graphical material.

[1] Recent summaries: a) T. Carell, L. T. Burgdorf, L. M. Kundu, M. Cichon, Curr. Opin. Chem. Biol. 2001, 5, 491-498; b) T. Lindahl, R.D. Wood, Science 1999, 286, 1897-1905; c) E. C. Friedberg, G.C. Walker, W. Siede, DNA Repair and Mutagenesis, American Society for Microbiology Press, Washington DC, 1995.

[2] Recent reviews and commentaries: a) E.C. Friedberg, P. L. Fischhaber, C. Kisker, Cell 2001, 107, 9-12; b) Z, Livneh, J. Biol. Chem. 2001, 276, 25639-25642; c) M. F. Goodman, B. Tippin, Nat. Rev. Mol. Cell. Biol. 2000, 1, 101 109; d) U. Hübscher, H.P. Nasheuer, J.E. Syvaoja, Trends Biochem. Sci. 2000, 25, $143-$ 147; e) E. C. Friedberg, W. J. Feaver, V.L. Gerlach, Proc. Natl. Acad. Sci. USA 2000, 97, $5681-5683$; f) E. C. Friedberg, V. L. Gerlach, Cell, 1999, 98, 413-416; g) R. E. Johnson, M.T. Washington, S. Prakash, L. Prakash, Proc. Natl. Acad. Sci. USA 1999, 96, 12224 12226.

[3] a) A. Vaisman, A. Tissier, E. G. Frank, M. F. Goodman, R. Woodgate, J. Biol. Chem. 2001,
276, 30615-30622; b) R.E. Johnson, M.T. Washington, L. Haracska, S. Prakash, L. Prakash, Nature 2000, 406, 1015-1019.

[4] K. Bebenek, A. Tissier, E. G. Frank, J. P. McDonald, R. Prasad, S. H. Wilson, R. Woodgate, T. A Kunkel, Science 2001, 291, 2156-2159.

[5] a) E. Glick, K. L. Vigna, L. A. Loeb, EMBO 2001, 20,7303-7312; b) M.T. Washington R. E. Johnson, L. Prakash, S. Prakash, Proc. Natl Acad. Sci. USA 2001, 98,8355-8360; c) R.E Johnson, M. T. Washington, S. Prakash, L. Prakash, J. Biol. Chem. 2000, 275, 74477450; d) C. Masutani, R. Kusumoto, S. Iwai, F Hanaoka, EMBO J. 2000, 19, 3100-3109 e) R. E. Johnson, C. M. Kondratick, S. Prakash L. Prakash, Science 1999, 285, 263-265; f) C Masutani, R. Kusumoto, A. Yamada, N. Dohmae, M. Yokoi, M. Yuasa, M. Araki, S. Iwai, K Takio, F. Hanaoka, Nature 1999, 399, 700-704; g) R. E. Johnson, S. Prakash, L. Prakash, Science 1999, 283, $1001-1004$

[6] a) L. F. Silvian, E. A. Toth, P. Pham, M. F. Good man, T. Ellenberger, Nat. Struct. Biol. 2001, 8 $984-989$; b) B.-L. Zhou, J. D. Pata, T. A. Steitz Mol. Cell 2001, 8, 427-437; c) J. Trincao, R.E Johnson, C. R. Escalante, S. Prakash, L. Prakash, A. K. Aggarwal, Mol. Cell 2001, 8, 417-426 d) H. Ling, F. Boudsocq, R. Woodgate, W. Yang, Cell 2001, 107, 91-102.

[7] Recent summaries: a) P.H. Patel, L. A. Loeb Nat. Struct. Biol. 2001, 8, 656-659; b) P.H Patel, M. Suzuki, E. Adman, A. Shinkai, L. A.
Loeb, J. Mol. Biol. 2001, 308, 823-837; c) T. A Kunkel, K. Bebenek, Annu. Rev. Biochem. 2000, $69,497-529$.

[8] a) M. C. Franklin, J. Wang, T. A. Steitz, Cell 2001 98, 413-416; b) S. Doublié, S. Tabor, A.M Long, C. C. Richardson, T. Ellenberger, Nature 1998, 391, 251-258; c) Y. Li, S. Korolev, G. Waksman, EMBO J. 1998, 17, 7514-7525 d) J. R. Kiefer, C. Mao, J. C. Braman, L. S. Beese Nature 1998, 391, 304-307; e) H. F. Huang, R Chopra, G. L. Verdine, S.C. Harrison, Science $1998,282,1669-1675 ;$ f) D. L. Ollis, P. Brick, R. Hamlin, N. G. Xuong, T. A. Steitz, Nature 1985 $313,762-766$.

[9] For DNA polymerases that are not handlike, see: a) A. K. Showalter, I.-J.L. Byeon, M.-I. Su, M.-D. Tsai, Nat. Struct. Biol. 2001, 8, 942-946 b) M. W. Maciejewski, R. Shin, B. Pan, A. Marintchev, A. Denninger, M. A. Mullen, K Chen, M. R. Gryk, G. P. Mullen Nat. Struct. Biol. 2001, 8, 936-941.

[10] a) E.T. Kool, J.C. Morales, K.M. Guckian, Angew. Chem. 2000, 112, 1046-1068; Angew. Chem. Int. Ed. 2000, 39, $991-1009$; b) T. A Kunkel, S. H. Wilson, Nat. Struct. Biol. 1998, 5 95-99; c) U. Diederichsen, Angew. Chem. 1998, 110, 1745-1747; Angew. Chem. Int. Ed. 1998, 37, 1655 -1657; d) M. F. Goodman, Proc Natl. Acad. Sci. USA 1997, 94, 10493-10495. 